\title{
Research on Influence of Flight Accuracy of Short-range Inertial Aircraft based on Control Theory and Mathematical Modelling
}

\author{
Wang Yan, Cai Jifei, Zhang Yang and Li Guang \\ Beijing Institute of Graphic Communication, Beijing, 102600, China \\ wangyanasda@126.com
}

\begin{abstract}
The short-range inertial aircraft has a short flight time, and its flight accuracy is influenced by various disturbance factors, and it is highly difficult to control the guidance. In this paper, various disturbance influences on the flight accuracy of ignoring after launching" aircraft is researched, through the mathematical modeling of such kind of aircraft and the mathematical modeling of various disturbance factors, the corresponding disturbance flight disturbance trajectory is analyzed under the influence of many disturbance factors like crosswind, initial disturbance and thrust eccentric, and it is concluded that the position control inertial guidance is a good plan, which can effectively eliminate the crosswind disturbance influence, then the engine thrust curve is relatively curve, the axial accelerometer can be omitted. For the above inertial guidance plan, the accuracy of angular position gyroscope on the aircraft is an important factor to decide the inertial guidance accuracy of the aircraft, thus providing key technical basis for the comprehensive evaluation of overall aircraft plan and the deep research of aircraft.
\end{abstract}

Keywords: Aircraft; Shoulder-type Soft Launch; Inertial Guidance; Flight Trajectory Simulation; Flight Accuracy

\section{Introduction}

Ignoring after launching short-range inertial guidance aircraft has a flight distance of only hundreds of meters, and flight time only several seconds, the launching process of shoulder-type soft launch aircraft is as shown in Figure 1 below. After discovering the target, the shooter starts the launch control equipment, aims at and traces the target, the launch control equipment records the angular speed of the target aiming line and calculates the average angular acceleration rate according to the nearly $2 \mathrm{~s}$ angular speed. When the shooter pulls the trigger and decides to launch, the launch control equipment sends a power-up signal, and sets the aiming line initial angular velocity and average angular acceleration rate to the inertial guidance system. When the inertial system is of attitude control plan, the launching control equipment also must set the wind speed information to the inertial guidance system, and the process of information setting by the launching control equipment to the aircraft can be completed by directly writing the data to the storage device in the aircraft. The launching plan of target scanning line information setting is superior to the launching plan of front light spot aiming, and it is characterized by simple operation and high launching precision etc. $[1,6,7,8]$.

According to the different projections of the aircraft flight trajectory in the horizontal plane and vertical plane, the ideal flight trajectory with the projection on the horizontal plane being a line is called straight flight trajectory, after launching, the aircraft with straight flight trajectory flies to the target along a straight line; and the ideal flight trajectory with a projection on the horizontal line being a curve is called curve flight trajectory. The straight flight trajectory launch control system calculates the horizontal angle of land required to hit the target before launch according to the aiming line 
information obtained by the shooter during aiming and tracking, and forms an aiming light spot, and the shooter should aim at the light spot again. For the curve flight trajectory, it is not needed to calculate the horizontal angle of lead before launching and the shooter directly launches the aircraft after aiming at and tracking the target, with a short aiming time and high requirements for the aircraft flight control system. Ignoring after launching short-range inertial guidance aircraft has a short flight time and the flight accuracy is greatly influenced by various disturbance factors. In this paper, various disturbance influences on the flight accuracy of "ignoring after launching aircraft is researched, and through modeling of the disturbance factors, the corresponding disturbance fight trajectory under various disturbance factors like crosswind, initial disturbance and thrust eccentric.
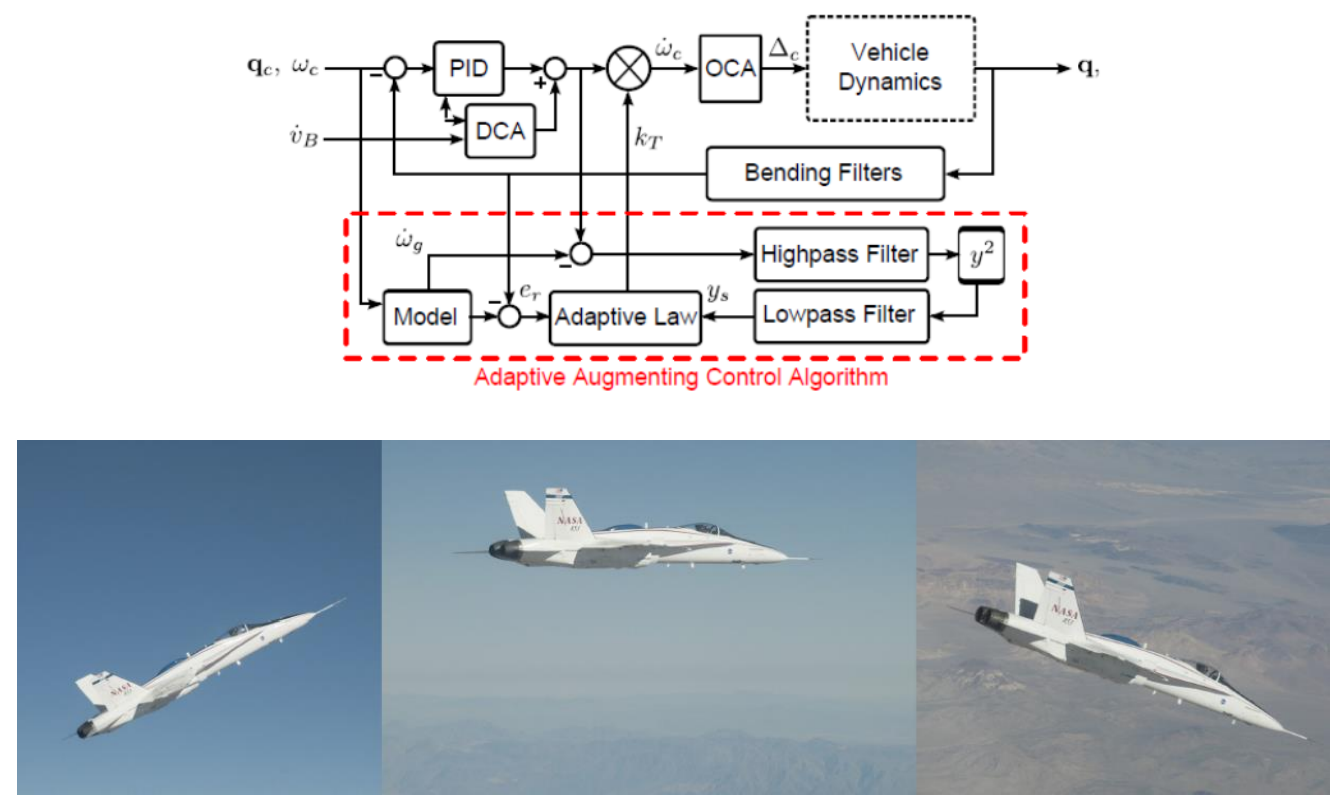

Figure 1. The Launching and Controlling Program in Setting and Launching Scheme of Line of Sight Information

\section{The Proposed Methodology}

\subsection{The Overview of the Approach}

The aerodynamic parameters of aircraft are calculated with MGAERO professional software. During the calculation of overall parameters of aircraft, the specific aerodynamic parameter of the aircraft and the structural mass of each subsystem are considered. To research the initial disturbance, the flight trajectory is calculated by combining the rigid body fight trajectory and particle flight trajectory. The non-control section flight trajectory after the aircraft goes out of the launch canister is calculated with rigid body flight trajectory, so as to accurately calculate the flight trajectory data at the starting control point. To simplify the problem, controlled particle flight trajectory is used to calculate the flight trajectory after controlling of aircraft. The flight trajectory simulation includes the non-control rigid body flight trajectory and controlled particle flight trajectory simulation. To research the initial disturbance, a small section of noncontrolled flight trajectory after the aircraft goes out of the launch canister is calculated with the simplified rigid body flight trajectory model, and at the same time, in order to simplify the problem, the flight trajectory after controlling of aircraft is calculated based on the controlled particle flight trajectory model. Based on the hypothesis of "transient 
equilibrium", by calculating the controlled particle flight trajectory, it is possible to get the "stable value" of the aircraft movement parameters $[2,9,10,11]$.

\subsection{The Kinematical Equation}

The centroid and attitude are respectively described in the flight trajectory coordinate system and quasi aircraft coordinate system, the influence of small $\gamma_{v}$ is ignored, the aircraft movement is respectively researched on the vertical plane and horizontal plane, including the kinematical equation of rolling and non-rolling aircrafts, as well as the kinematical equation of non-controlled rigid body and controlled particle, and the standard aircraft kinematical equation is adopted in the formula and parameters, here no detailed explanation is conducted [2-4].

The ideal flight trajectories of horizontal plane of aircraft include the curve flight trajectory and straight flight trajectory, and the controlled aircraft curve flight trajectory including the import section and guidance section [12-14]. The ideal flight trajectory of horizontal plane is as shown in Figure 2.

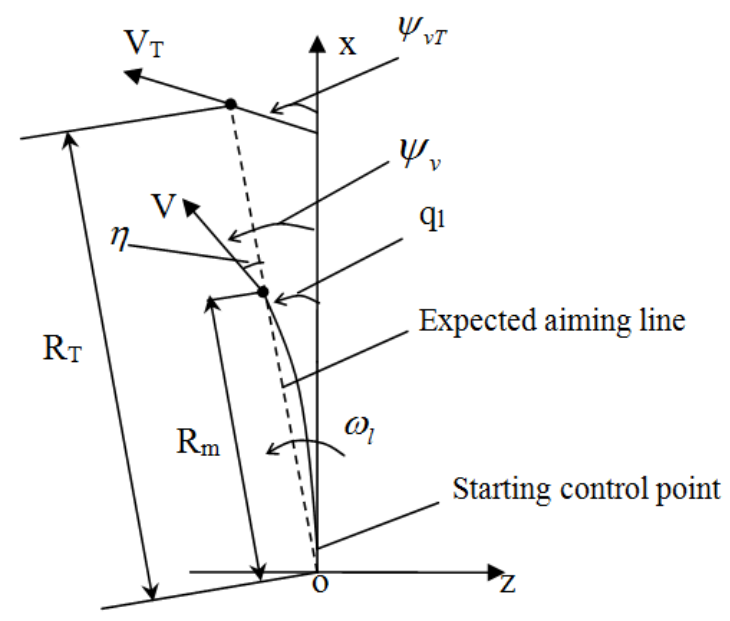

Figure 2. The Ideal Flight Trajectory Chart of Horizontal Plane

Imagine that there is always a horizontal aiming line from the shooter to the target in the process of flight of aircraft, and as long as the aircraft is controlled to fly along the target airline, the target can be hit. Let that the rotating angular speed of the initially set target aiming line of aircraft is $\omega_{l 0}$, initially set angular acceleration rate is $\varepsilon_{l 0}$, and the target aiming line is of approximate constant acceleration movement, and the horizontal rotation angular speed and angle are:

$$
\begin{aligned}
& \omega_{l}=\omega_{l 0}+\varepsilon_{l 0} t \\
& q_{l}=\omega_{l 0} t+\frac{1}{2} \varepsilon_{l 0} t^{2}
\end{aligned}
$$

To make the aircraft always on the target air-line, the following equation should be met:

$$
\begin{gathered}
R_{m} \frac{d q_{l}}{d t}=V \sin \eta \\
\frac{d R_{m}}{d t}=V \cos \eta \\
\psi_{v}=q_{l}+\eta
\end{gathered}
$$


In the formula above, $R_{m}, \eta, q_{l}, \omega_{l}, \varepsilon_{l}$ respectively represent the aircraft flight distance along the target aiming line, included angle between aircraft speed direction and target aiming line, rotation angle of target aiming line, rotation angular speed of target aiming line and rotation angular acceleration.

\subsection{Modeling of Disturbance Factors}

In the following analysis, rolling plan is adopted, therefore, the influence of engine thrust eccentric can be basically ignored. In addition, the main engine thrust eccentric an actual thrust error can be guaranteed to be small generally, therefore, the main engine thrust change does not have a great influence on the hitting accuracy of aircraft, and the computer in the aircraft can calculate the axial acceleration speed according to the thrust curve, so as to save the axial accelerometer.

\subsubsection{Main Factors for System Error}

The measuring error of ground angular rate gyroscope will cause inaccurate initial setting of aircraft, so as to influence the aircraft hitting accuracy. The angular rate gyroscope has a low resolution, which will not be sensitive to the movement of low-speed target, and the angular rate gyroscope has a poor nonlinearity, which will cause inaccurate measuring information. The influence of angular rate gyroscope resolution on the aircraft hitting accuracy can be calculated from the two formulas below [4-12].

$$
\begin{aligned}
V_{T \text { min }} & =S_{T} R_{l} \pi / 180 \\
\Delta z & =V_{T \text { min }} t_{T}
\end{aligned}
$$

In the formula above, $V_{T \text { min }}$ is the minimum sped of target sensitive to angular rate gyroscope, $R_{l}$ is the angular rate gyroscope resolution, $S_{T}$ is the target distance, $t_{T}$ is the aircraft flight time, and $\Delta z$ is the transverse hitting error of aircraft.

As there is the nonlinearity for the angular rate gyroscope, the relation between the measured value and actual value of the angular rate is as follows:

$$
\omega_{l c e}=\omega_{l 0}\left(1+N_{l}\right)
$$

\subsubsection{Angular Position Gyroscope on Aircraft}

The measuring error of angular position gyroscope on the aircraft makes the measured value of the attitude angle different from the actual value, thus influencing the control accuracy of the inertial guidance system. When it is found that the gyroscope error changed in an approximately linear relation, the relation between the measured value of attitude angle and the actual value is as follows [13-17].

$$
\begin{gathered}
\psi_{c e}=\psi+\Delta \xi_{o}+\xi_{o} t \\
\vartheta_{c e}=\vartheta+\Delta \xi_{i}+\xi_{i} t
\end{gathered}
$$

In the above formula, $\Delta \xi_{o}$ is the initial error of external frame of gyroscope, $\Delta \xi_{i}$ is the initial error of internal frame of gyroscope, $\xi_{0}$ is the drifting rate of external frame of gyroscope and $\xi_{i}$ is the drifting rate of external frame of gyroscope.

In addition to the initially set angular rate, the factor influencing the calculated accuracy of ideal flight trajectory deflection also include $\varepsilon_{l 0}, \dot{V}, V$, x etc., the $\dot{V}, V$ and $\mathrm{x}$ obtained based on the calculation of ideal flight trajectory will introduce error, and it is possible to research parameters such as $\dot{V}, V$ and $\mathrm{x}$ by researching the influence of thrust error on the flight trajectory of aircraft. $\varepsilon_{l 0}$ is used for certain control compensation 
to the target when there is advanced movement, and the $\varepsilon_{l 0}$ obtained based on differential calculation will introduce the setting error, let that the distance during target launching is $S_{T}$, horizontal speed is $V_{T z}$ and advanced speed is $V_{T x}$, then the angular rate of target aiming line $2 \mathrm{~s}$ before the launching is:

$$
\varepsilon_{l 2}=-\frac{2 V_{T z} V_{T x}}{\left(S_{T}-2 V_{T x}\right)^{2}}
$$

\subsubsection{Main Factors Generating Disturbance Error}

The component of the crosswind in the flight trajectory coordinate system is approximated to be:

$$
\left[\begin{array}{l}
W_{x 2} \\
W_{y 2} \\
W_{z 2}
\end{array}\right]=\left[\begin{array}{l}
-W_{z} \cos \theta \sin \psi_{v} \\
W_{z} \sin \theta \sin \psi_{v} \\
W_{z} \cos \psi_{v}
\end{array}\right]
$$

Relative speed of aircraft is:

$$
V_{w}=\sqrt{\left(V-W_{x 2}\right)^{2}+W_{y 2}^{2}+W_{z 2}^{2}}
$$

Additional sideslip angle:

$$
\Delta \beta_{w}=-\sin ^{-1}\left(W_{z 2} / V_{w}\right)
$$

Disturbing force and disturbing torque caused by crosswind:

$$
\begin{aligned}
& Z_{w}=C_{z}^{\beta} \Delta \beta_{w} q S \\
& M_{y w}=m_{y}^{\beta} q S L \Delta \beta_{w}
\end{aligned}
$$

\subsection{Initial Disturbance}

Influenced by the trembling of shooter during launch of launcher and the gap existing in launch canister, the aircraft will generate initial disturbance at the moment when it departs from the launch canister, the initial disturbance mainly includes the initial pitch angle deviation $\Delta \vartheta_{0}$, initial yaw angle deviation $\Delta \psi_{0}$, initial pitch angle speed deviation $\Delta \omega_{z 0}$, initial yaw angle speed deviation $\Delta \omega_{y 0}$, initial flight trajectory inclined angle deviation $\Delta \theta_{0}$ and initial flight trajectory deflection deviation $\Delta \psi_{v 0}$. The method to search the initial disturbance is to introduce the initial disturbance into the simulation initial value condition of the flight trajectory.

\section{The Flight Disturbance Factor Simulation}

\subsection{The Angular Rate Gyroscope}

When the target distance $S_{T}=600 \mathrm{~m}$, advanced speed $V_{T x}=0$ and the aircraft control plan is attitude control plan, the calculated result of flight trajectory of the angular rate gyroscope under different resolution conditions is as shown in Table 1.

In the table, $R_{l}$ represents the resolution of angular rate gyroscope, $V_{T z \min }$ represents the minimum horizontal speed of the target to be detected by the angular rate gyroscope, $\Delta z_{\text {end }}$ represents the z-direction flight deviation of aircraft caused by the resolution of angular rate gyroscope. 


\section{Table 1. The Influence of Angular Rate Gyroscope Resolution on Aircraft Hitting Rate}

\begin{tabular}{|l|l|l|}
\hline$R_{l}$ & $V_{T z \min }$ & $\Delta z_{\text {end* }}$ \\
\hline 0.005 & 0.052 & 0.139 \\
\hline 0.01 & 0.104 & 0.278 \\
\hline 0.05 & 0.520 & 1.39 \\
\hline
\end{tabular}

When the target distance $S_{T}=600 \mathrm{~m}$, horizontal speed $V_{T z}=15 \mathrm{~m} / \mathrm{s}$, advanced speed $V_{T x}=0$ and aircraft control plan is attitude control plan, the calculated result of flight trajectory of the angular rate trajectory under different nonlinear condition is as shown in Table 2.

In the table, $N_{l}$ represents the nonlinearity of angular rate gyroscope, and other symbols are described as above.

Table 2. The Influence of Angular Rate Gyroscope Nonlinearity on Aircraft Hitting Rate

\begin{tabular}{|l|l|l|l|}
\hline$N_{l}$ & $z_{\text {Tend }}$ & $z_{\text {end }}$ & $\Delta z_{\text {end }}$ \\
\hline 0 & 39.85 & 39.95 & 0.11 \\
\hline $0.5 \%$ & 39.85 & 40.15 & 0.31 \\
\hline $1 \%$ & 39.85 & 40.35 & 0.51 \\
\hline
\end{tabular}

\subsection{The Angular Position Gyroscope}

When the inertial guidance system is of attitude control plan, the flight trajectory curve when the gyroscope initial error is $0.1^{\circ}$ and external frame drifting changes from $-0.2 \%$ to $0.2^{\circ} \mathrm{s}$ is as shown in Figure 3, and the and the flight trajectory curve when the trajectory vertical initial error is $0.1^{\circ}$, internal frame drifting changes from $-0.2^{\circ} / \mathrm{s}$ to $0.2^{\circ} / \mathrm{s}$ is as shown in Figure 4. When the inertial system is of position control plan, the flight trajectory curve when the gyroscope horizontal initial error is $0.1^{\circ}$ and external frame drifting changes from $-0.2^{\circ} \mathrm{s}$ to $0.2^{\circ} \%$ is as shown in Figure 5, and the flight trajectory curve when the gyroscope vertical initial error is $0.1^{\circ}$ and internal frame drifting changes from $-0.2 \%$ to $0.2 \%$ is as shown in Figure 6 .

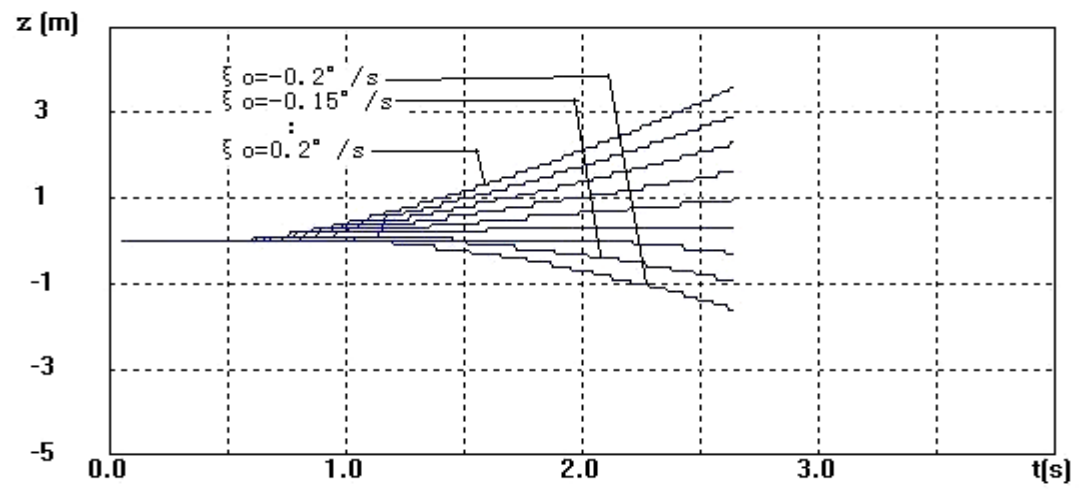

Figure 3. The Flight Trajectory Curve Under the Action of Horizontal Gyroscope Error (Attitude Control) 


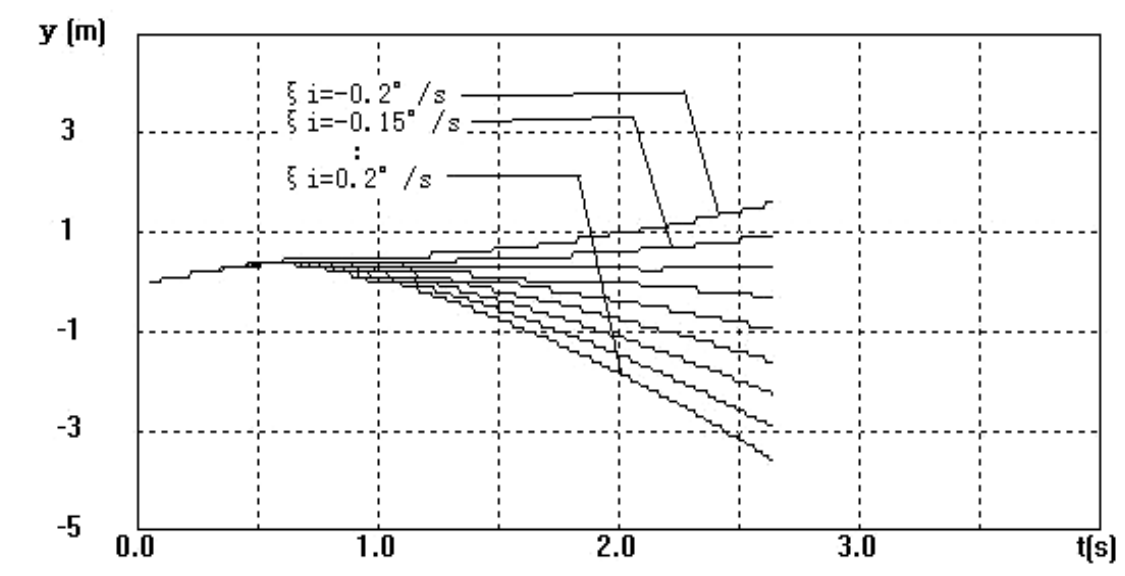

Figure 4. The Flight Trajectory Curve Under the Action of Vertical Gyroscope Error (Attitude Control)

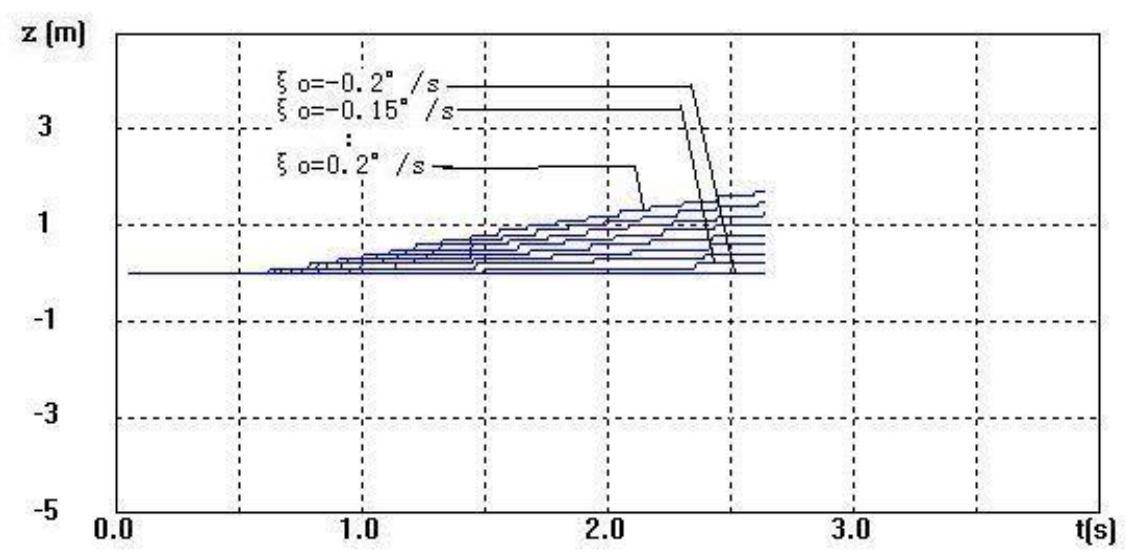

Figure 5. The Flight Trajectory Curve Under the Action of Horizontal Gyroscope Error (Position Control)

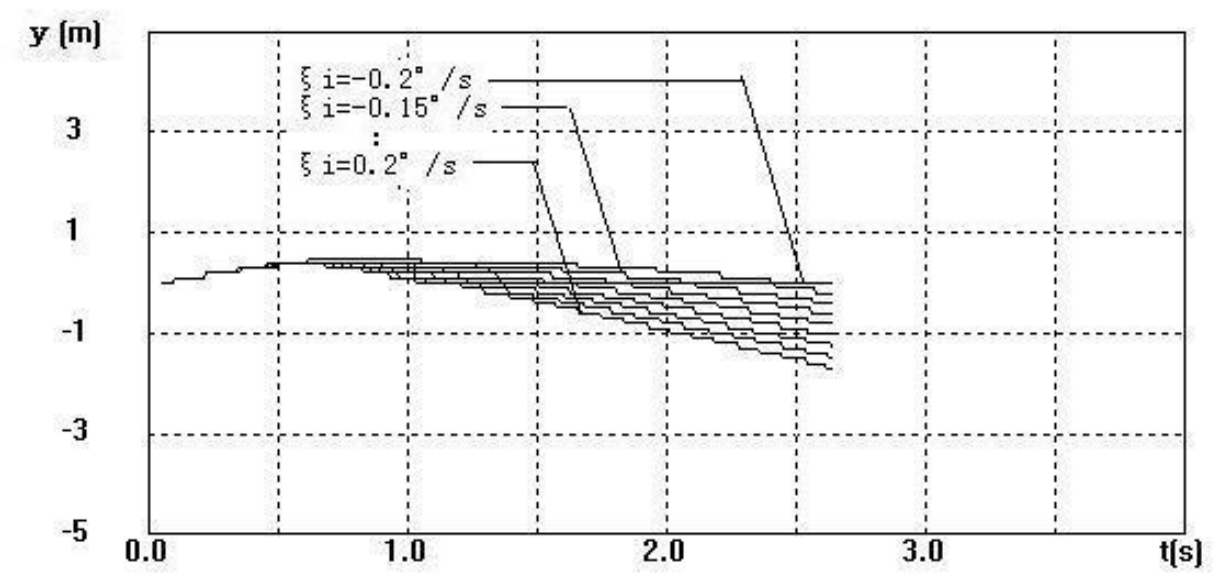

Figure 6. The Flight Trajectory Curve Under the Action of Vertical Gyroscope Error (Position Control) 


\subsection{The Calculation Model}

When the target distance $S_{T}=600 \mathrm{~m}$, horizontal speed $V_{T z}=15 \mathrm{~m} / \mathrm{s}$, advanced speed $V_{T x}=-15 \mathrm{~m} / \mathrm{s}$, the set angular rate changes from $0.00113-0.00125 \mathrm{arc} / \mathrm{s} 2$, and when the aircraft control plan is attitude control plan, the calculated result of flight trajectory under different set angular acceleration rates is as shown in Figure 3.

In the table, $\varepsilon_{l}$ represents the set angular accelerate rate of aircraft, and other symbols are described as above Table 3. The Influence of Nonlinearity of Angular Rate Gyroscope on the
Hitting Accuracy of Aircraft

\begin{tabular}{|l|l|l|l|}
\hline$\varepsilon_{l}$ & $z_{\text {Tend }}$ & $z_{\text {end* }}$ & $\Delta z_{\text {end* }}$ \\
\hline 0 & 37.64 & 35.38 & -2.26 \\
\hline 0.00113 & 37.66 & 37.44 & -0.22 \\
\hline 0.00119 & 37.66 & 37.55 & -0.11 \\
\hline 0.00125 & 37.66 & 37.66 & 0.00 \\
\hline
\end{tabular}

\subsection{The Influence of Crosswind}

When the target distance $S_{T}=600 \mathrm{~m}$, horizontal speed $V_{T z}=0 \mathrm{~m} / \mathrm{s}$, advanced speed $V_{T x}=0$ and when the aircraft control plan is attitude control plan, aimed at the two situations of having blast indicator and having no blast indicator, the result of flight trajectory when the crosswind speed is $10 \mathrm{~m} / \mathrm{s}$ and action time changes. When blast indicator is used for wind compensation, and when the crosswind speed is $10 \mathrm{~m} / \mathrm{s}$ and action time changes from 0 to $3 \mathrm{~s}$, the flight trajectory is as shown in Figure 7.

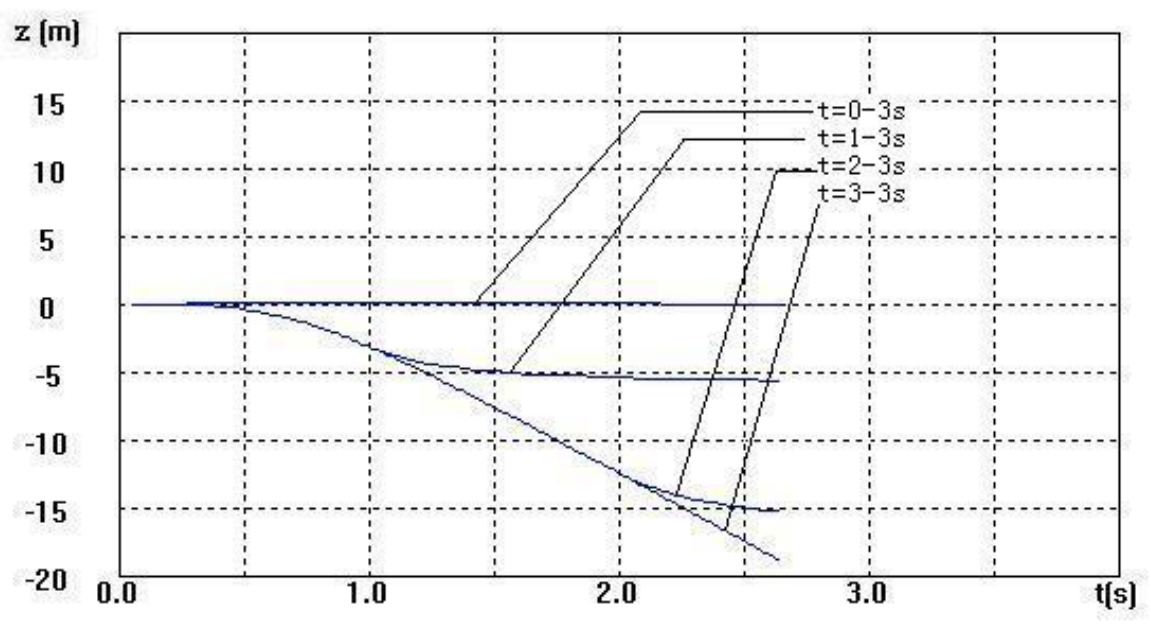

Figure 7. Flight Trajectory Curve of Under Crosswind Action in Different Action Time

When the target distance $S_{T}=600 \mathrm{~m}$, horizontal speed $V_{T z}=0 \mathrm{~m} / \mathrm{s}$, advanced speed $V_{T x}=0$, and when the aircraft control plan is attitude control plan, the flight trajectory under the initial disturbance of horizontal plane and vertical plane are calculated.

When the initial yaw angle deviation is $1^{\circ}$, initial yaw angle angular rate is 0 , and initial flight trajectory deflection error changes from $-1 \% \mathrm{~s}$ to $1 \% \mathrm{~s}$, the flight trajectory 
curve is as shown in Figure 8; when the initial pitch angle is $1^{\circ}$, initial pitch angle angular rate error is 0 and initial flight trajectory inclined angle changes from $-1 \% \mathrm{~s}$ to $1 \% \mathrm{~s}$ the flight trajectory curve is as shown in Figure 9.

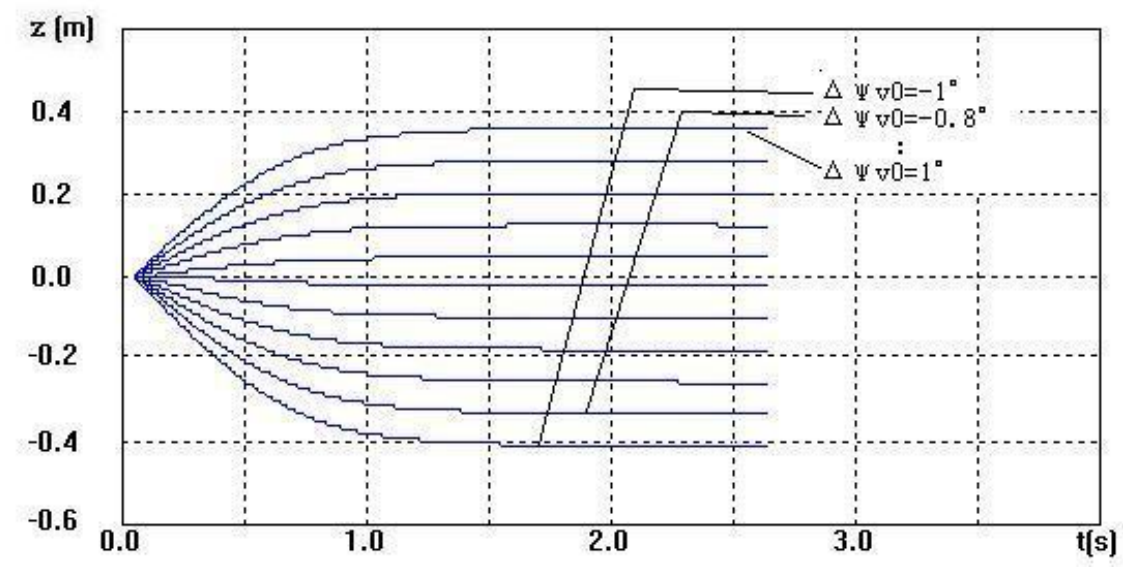

Figure 8. Flight Trajectory Curve Under Horizontal Initial Error

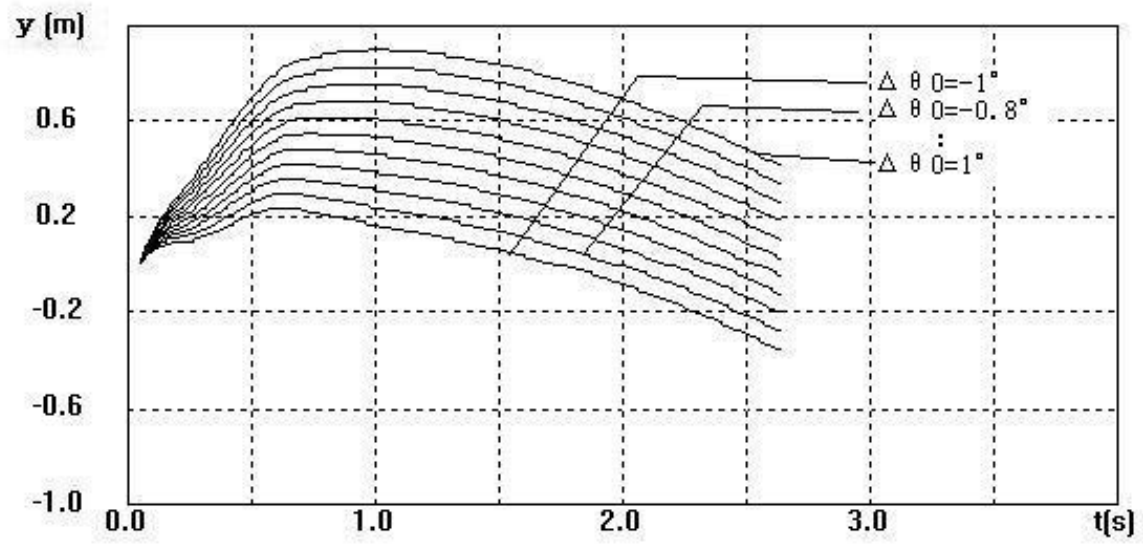

Figure 9. Flight Trajectory Curve Under Vertical Initial Error

\section{Experimental Simulation Result Analysis}

The higher the resolution of piezoelectric angular gyroscope is, the smaller the hitting accuracy of aircraft will be. Upon further simulation of flight trajectory, it can be concluded that to make the hitting error of aircraft caused by the resolution of angular rate gyroscope less than $0.5 \mathrm{~m}$, the resolution should be less than $0.018 \%$. The smaller the nonlinearity of piezoelectric angular gyroscope is, the smaller the hitting accuracy of aircraft will be. Upon further simulation of flight trajectory, it can be concluded that to make the hitting error of aircraft caused by the nonlinearity of angular rate gyroscope less than $0.5 \mathrm{~m}$, the nonlinearity should be less than $1 \%$.

The increasing of gyroscope error will reduce the hitting accuracy of aircraft, especially, the increasing of error of vertical gyroscope will make the aircraft contact with the ground, the gyroscope with small gyroscope initial error and drifting should be used for the inertial guidance system. When the inertial guidance system is of position control plan, the influence of gyroscope error on the hitting accuracy of aircraft is smaller than 
using the attitude control plan, so the requirement for the gyroscope accuracy can be reduced slightly. Upon further simulation of flight trajectory, it can be concluded that when the inertial guidance system is of attitude control plan, to make the hitting error of aircraft caused by the initial error of angular position gyroscope less than $0.5 \mathrm{~m}$, the initial error should be less than $0.05^{\circ}$, and to make the hitting error of aircraft caused by the drifting of gyroscope less than $0.5 \mathrm{~m}$, the external frame drifting should be less than $0.04 \%$; when the inertial system is of position control plan, to make the hitting error of aircraft caused by the initial error of angular position gyroscope less than $0.5 \mathrm{~m}$, the initial error should be less than $0.045^{\circ}$, and to make the hitting error of aircraft caused by the drifting of gyroscope less than $0.5 \mathrm{~m}$, the external frame drifting should be less than $0.12 \%$ s.

When the target has advanced movement, the setting of angular acceleration has a significant compensation action, which greatly improves the hitting accuracy of aircraft, and the change of aircraft set angular acceleration rate caused by the calculation error has a very small influence on the hitting accuracy of aircraft, therefore, it is feasible to improve the hitting accuracy of aircraft by setting the average angular acceleration rate.

To make the error of aircraft hitting accuracy caused by the error of angular trajectory deflection less than $0.5 \mathrm{~m}$, the flight trajectory deflection shall have an initial error less than $1.25^{\circ}$, and to make the error of aircraft hitting accuracy caused by the initial error of flight trajectory inclined angle less than $0.5 \mathrm{~m}$, the flight trajectory inclined angle shall have an initial error less than $1.25^{\circ}$. The initial disturbance does not have a great influence on the hitting accuracy of aircraft, mainly because the aircraft maintains the flight trajectory angle by maintaining an ideal attitude angle during flight. Therefore, for the short-range aircraft with attitude control plan, the influence of trembling of shooter during launching nay not be considered basically.

When the aircraft is of attitude control inertial guidance plan, it is required to use the rolling plan to eliminate the influence of thrust eccentric. Although the crosswind disturbance can be compensated and modified in a certain manner by removing the blast indicator, it is unable to completely eliminate the influence of crosswind, and the change of crosswind has a great influence on the hitting accuracy of aircraft.

\section{Conclusion and Summary}

Through the analysis on influence of various disturbance factors on the flight trajectory of soft launch short-range aircraft, it is concluded that the position control inertial guidance plan is a good plan, which can effectively eliminate the influence of crosswind disturbance, when the engine thrust curve is relatively curve, the axial accelerometer can be omitted. For the above inertial guidance plan, the accuracy of angular position gyroscope on the aircraft is an important factor to decide the inertial guidance accuracy of the aircraft.

\section{Acknowledgements}

This work was supported by these projects: PXM2015-014223-000007; KM201510015005; TJSHG201510015011; 23190114014

\section{References}

[1] C. A. Woolsey, "Review of marine control systems: Guidance, navigation, and control of ships, rigs and underwater vehicles", Journal of Guidance, Control and Dynamics, vol. 28, no. 3 (2005), pp. 574-575.

[2] S. A. Nicolau, L. Goffin and L.Soler, "A low cost and accurate guidance system for laparoscopic surgery: Validation on an abdominal phantom", Proceedings of the ACM symposium on Virtual reality software and technology. ACM, (2005).

[3] D.T. McRuer, G. Dunstan and A. Irving, "Aircraft dynamics and automatic control", Princeton University Press, (2014). 
[4] N. Barbour and G. Schmidt, "Inertial sensor technology trends", Sensors Journal, IEEE 1.4 (2001), pp. 332-339.

[5] B. Shuang, "Improved analysis for determining diffusion coefficients from short, single-molecule trajectories with photoblinking", Langmuir, vol. 29, no. 1, (2012), pp. 228-234.

[6] R. Du, "Design of three-dimensional nonlinear guidance law with bounded acceleration command", Aerospace Science and Technology, vol. 46, (2015), pp. 168-175.

[7] S. Sun, D. Zhou and W.-T. Hou, "A guidance law with finite time convergence accounting for autopilot lag”, Aerospace Science and Technology, vol. 25, no. 1, (2013), pp. 132-137.

[8] H. Song, "Integrated Design of Interceptor Guidance and Control Based on L1 Adaptive Control", Intelligent Human-Machine Systems and Cybernetics (IHMSC), 2013 5th International Conference on, IEEE, vol. 1, (2013).

[9] S. Sun, D. Zhou and W.-T. Hou, "A guidance law with finite time convergence accounting for autopilot lag", Aerospace Science and Technology, vol. 25, no. 1, (2013), pp. 132-137.

[10] V. Nekoukar and A. Erfanian, "Adaptive fuzzy terminal sliding mode control for a class of MIMO uncertain nonlinear systems", Fuzzy Sets and Systems,vol. 179, no. 1, (2011), pp. 34-49.

[11] Neila, M. B. Romdhane and D. Tarak, "Adaptive terminal sliding mode control for rigid robotic manipulators", International Journal of Automation and Computing, vol. 8, no. 2, (2011), pp. 215-220.

[12] D. Zhao and T.Zou, "A finite-time approach to formation control of multiple mobile robots with terminal sliding mode", International Journal of Systems Science, vol. 43, no 11, (2012), pp. 1998-2014.

[13] K. Xu, and C. Mou, "Terminal sliding mode control with disturbance observer for autonomous mobile robots", Control Conference (CCC), 2015 34th Chinese. IEEE, (2015).

[14] M. Sanjoy and C. Mahanta, "Adaptive second order terminal sliding mode controller for robotic manipulators", Journal of the Franklin Institute, vol. 351, no. 4, (2014), pp. 2356-2377.

[15] L. Yang and J. Yang, "Nonsingular fast terminal sliding-mode control for nonlinear dynamical systems", International Journal of Robust and Nonlinear Control, vol. 21, no. 16, (2011), pp. 1865-1879.

[16] L. Córdoba, Diego and R. Ortega, "An Observer-Based Scheme for Decentralized Stabilization of Large-Scale Systems With Application to Power Systems", Asian Journal of Control, vol. 17, no. 1 (2015), pp. 124-132.

[17] Song and Haitao, "Integrated Design of Interceptor Guidance and Control Based on L1 Adaptive Control", Intelligent Human-Machine Systems and Cybernetics (IHMSC), 2013 5th International Conference on, IEEE, vol. 1, (2013).

[18] Gharib, M. Reza and M. Movenian, "Synthesis of robust PID controller for controlling a single input single output system using quantitative feedback theory technique”, Scientia Iranica. Transaction B, Mechanical Engineering, vol. 21, no. 6, (2014), pp. 1.

[19] Dickinson and Benjamin, "Robust and adaptive control of a rocket boosted missile", American Control Conference (ACC), IEEE, 2015, (2015).

[20] Azizi and Askar, "New robust feedback linearization method based on nonlinear disturbance observer", Industrial Electronics (ISIE), 2014 IEEE 23rd International Symposium on. IEEE, (2014). 
International Journal of Control and Automation Vol. 9, No. 12 (2016) 